\title{
Concept of a Movable Flat Panel Detector for X-ray Imaging during Surgical Interventions
}

\author{
S. Engel ${ }^{1}$, M. Käseberg ${ }^{1}$, F. Stopp ${ }^{2}$, F. Fehlhaber ${ }^{1}$, E. Keeve ${ }^{1,2}$ \\ ${ }^{1}$ Fraunhofer-Institute for Production Systems and Design Technology IPK, Berlin, Germany \\ ${ }^{2}$ Charité - Universitätsmedizin Berlin, Berlin, Germany \\ Contact:keeve@charite.de
}

\section{Introduction}

For better suited two- and three-dimensional intraoperative imaging a new approach of an x-ray imaging system with uncoupled x-ray source and x-ray detector is presented. This system benefits from a free positioning of both modules which results in new opportunities for x-ray imaging. In order to provide a moveable imaging sensor a new concept for a patient-near flat panel detector is presented.

\section{Methods}

The goal was a conceptual device moving a flat panel detector integrated to the operation room table. The optimization criteria focus on the possible image quality and diagnostic potentials for the surgeon by using this device. Additionally two major requirements were considered: the possibility of lateral projections and the optimization of the reconstructed volume size. To achieve a large reconstruction volume it is planned to position the detector near to the patient.

\section{Results}

The concept to move the X-ray flat panel uses an at least three-axis robotic arm. The arm is located under the OR-table surface and typically attached to the table base. With a length of each arm part of at least half the table width the flat panel can be positioned directly below and beside the patient. Because the flat panel is moving near the table the robot control needs to know the exact position and measures of the table to plan trajectories and avoid collisions.

\section{Conclusion}

A flat panel concept that enables new possibilities for integrated x-ray imaging systems during surgery procedures has been designed. Our approach includes a moveable flat panel detector device attached to the table base which is able to provide open access to the patient before, during and after the surgery.

\section{Acknowledgement}

This work is funded by the German Federal Ministry of Education and Research (BMBF), research grant $01 \mathrm{EZ1115.}$ 